\title{
Route repetition and route retracing: effects of cognitive aging
}

\author{
Jan M. Wiener *, Hana Kmecova and Olivier de Condappa
}

Psychology Research Centre, Bournemouth University, Poole, UK

Edited by:

Thomas Wolbers, University

of Edinburgh, UK

Reviewed by:

Denise Head, Washington

University in St. Louis, USA

John Philbeck, The George

Washington University, USA

*Correspondence:

Jan M. Wiener, Psychology

Research Centre, Bournemouth

University, Poole House, Talbot

Campus, Fern Barrow, Poole,

Dorset BH12 5BB, UK.

e-mail: jwiener@bournemouth.

ac.uk
Retracing a recently traveled route is a frequent navigation task when learning novel routes or exploring unfamiliar environments. In the present study we utilized virtual environments technology to investigate age-related differences in repeating and retracing a learned route. In the training phase of the experiment participants were guided along a route consisting of multiple intersections each featuring one unique landmark. In the subsequent test phase, they were guided along short sections of the route and asked to indicate overall travel direction (repetition or retracing), the direction required to continue along the route, and the next landmark they would encounter. Results demonstrate age-related deficits in all three tasks. More specifically, in contrast to younger participants, the older participants had greater problems during route retracing than during route repetition. While route repetition can be solved with egocentric response or route strategies, successfully retracing a route requires allocentric processing. The age-related deficits in route retracing are discussed in the context of impaired allocentric processing and shift from allocentric to egocentric navigation strategies as a consequence of age-related hippocampal degeneration.

Keywords: route retracing, route learning, cognitive aging, spatial memory, wayfinding

\section{INTRODUCTION}

Age-related differences in navigation abilities are well established both in animal and human literature (Rosenzweig and Barnes, 2003; Moffat, 2009). While age-related performance declines have been reported for different navigation tasks, they seem to be particularly pronounced in unfamiliar environments (Devlin, 2001). One navigation ability crucial for navigating unfamiliar environments is retracing-navigating a recently traveled route from the end to the start-as it enables a navigator to return to a known part of the environment (Lorenz, 1952; Miller and Eilam, 2011). Specific age-related deficits in route retracing would increase the risk of getting lost when navigating novel environments and could thus explain why older adults often report avoiding unfamiliar places and routes (Burns, 1999). While route retracing is a common navigation task, especially when exploring unfamiliar environments, it has received surprisingly little attention in the literature and it is unclear how it is accomplished. In the current study we present a novel experimental paradigm to investigate the cognitive processes and strategies involved in route learning and route retracing in more detail. Moreover, by comparing route retracing performance between a younger and older age group we investigate the effects of cognitive aging on route retracing.

Route knowledge is typically conceptualized as a series of stimulus-response associations (Trullier et al., 1997). A single stimulus-response association consists of recognizing the current place (e.g., by recognizing a landmark associated with that place) and selecting the direction in which to proceed along the route (Waller and Lippa, 2007). Learning a route with multiple decision points, therefore, requires knowledge of landmarks along the routes, associations of directional information with these landmarks, and knowledge about the order of landmarks.

Note that route knowledge that takes the form of stimulusresponse pairs is inherently uni-directional, allowing a route to be repeated. The stimulus-response pairs are encoded in an egocentric reference frame during learning ("Turn left at X"). During route retracing, however, the decision points are approached from a viewpoint different to that experienced before. Accordingly, the egocentric stimulus-response pairs that were encoded during route learning do not support route retracing. Route retracing requires knowledge about the spatial relationship between the direction from which a decision point is approached and the direction in which the route proceeded. Such a representation is viewpoint independent-i.e., allocentric - and would support for route retracing.

Egocentric and allocentric navigation strategies are supported by different neuronal circuits: egocentric strategies involve the parietal cortex and the caudate nucleaus, while allocentric strategies are hippocampus dependent (McDonald and White, 1994; Wolbers et al., 2004; Burgess, 2008). While cognitive aging affects egocentric strategies (Barrash, 1994; Wilkniss et al., 1997; Moffat et al., 2001; Head and Isom, 2010), allocentric strategies seem to be more severely affected (Begega et al., 2001; Moffat and Resnick, 2002). As a result, a number of studies report shifts from allocentric to egocentric strategies with increasing age (Barnes et al., 1980; Nicolle et al., 2003; Rodgers et al., 2012; Wiener et al., under review). Age-related hippocampal degeneration offers an explanation for the impaired allocentric processing and according strategy shifts (Raz et al., 2010). 
In the current study we used a novel experimental paradigm to investigate the cognitive processes and strategies involved in route retracing. Specifically, we tested the hypothesis that route retracing, in contrast to route repetition, relies on allocentric processing. In the experiment, participants were navigated along a complex route with multiple decision points each featuring a unique landmark. In the subsequent test phase they were guided along sections of the route either in the direction of original travel (route repetition) or in the opposite direction (route retracing). Their tasks were, first, to indicate travel direction (route repetition or route retracing), second to indicate the direction required to continue along the route to either reach the end or the start place (depending on travel direction), and third, to identify the next landmark encountered on route if the current travel direction was maintained. Comparisons of performance in these tasks between route repetition and route retracing trials allowed for first insights into the nature of route retracing. In order to test whether route retracing in fact involves allocentric processing, we compared performance between a younger and older participant group. In line with earlier research into the effects of cognitive aging on navigation abilities (Moffat et al., 2001; Head and Isom, 2010), we expected an overall effect of age on performance in all three tasks. If route retracing - as argued above-relied on allocentric processing, we expected additional age-related performance declines for route retracing trials as compared to route repetition trials reflecting the particularly adverse effects of cognitive aging on allocentric navigation strategies (Moffat and Resnick, 2002; Iaria et al., 2009).

\section{MATERIALS AND METHODS PARTICIPANTS}

Forty participants [20 younger (eight females; mean age $20.53 \pm$ 1.84 years, range $25-30$ ); 20 older (11 females; mean age $69.45 \pm$
5.48 years, range 61-85)] took part in the experiment. The montreal cognitive assessment (MoCA; Nasreddine et al., 2005) was administered to all participants to screen for mild cognitive impairment (MCI). No participant had to be excluded based on the recently recommended MoCA cut-off score for the MCI of 23 (Luis et al., 2009). The average MoCA scores for the young and old age group were 27.56 (young) and 27.65 (old).

\section{THE VIRTUAL ENVIRONMENT}

Using Vizard 3.0 (WorldViz) we created a virtual route consisting of 11 four-way intersections (see Figure 1). Each intersection could be identified by a unique single landmark - an image of an object - that was mapped onto a cube suspended from the wall. During the experiment, participants were passively transported along the entire route in the training phase and along parts of the route in the test phase.

\section{PROCEDURE}

The experiment consisted of six experimental sessions. Each experimental session was composed of a training phase and a test phase. In the training phase, participants were transported along the entire route twice. Their task was to memorize the route. The test phase consisted of 18 trials, each of which was composed of three tasks:

- Route Direction Task. First, participants were transported along two intersections of the original route either in the same direction as during training (route repetition, nine trials) or in the opposite direction (route retracing, nine trials). For example, in a route repetition trial they would approach the intersection with the sofa, turn left and then approach the intersection with the football where movement stopped (see Figure 1). Participants were asked to indicate the direction of

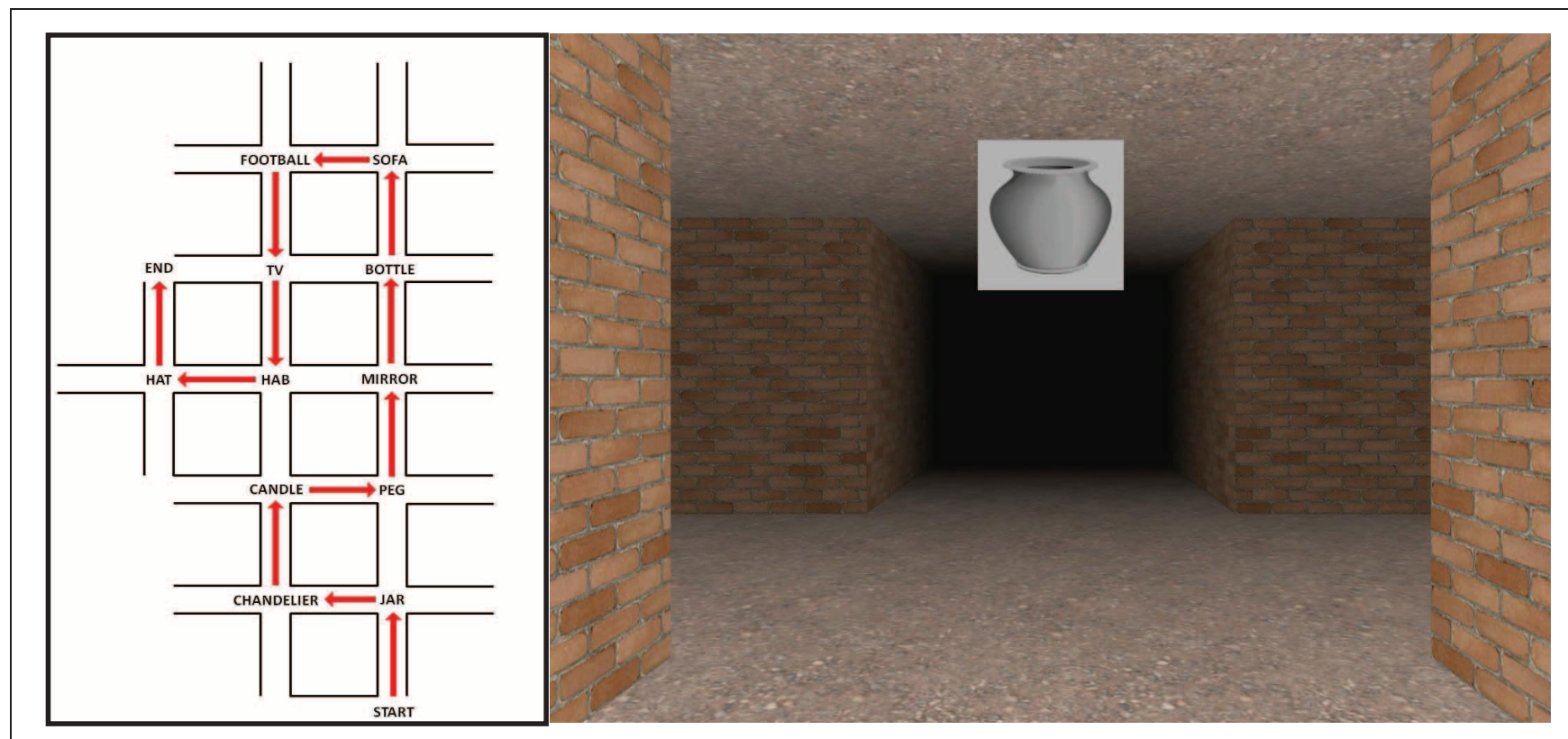

FIGURE 1 | Left: schematic drawing of the route consisting of 11 intersections; right: screen-shot of one of the intersections. 
travel (repetition or retrace). Participants were instructed to respond as soon as they identified the travel direction, even if this occurred during the actual movement. Chance level for this task was $50 \%$.

- Intersection Direction Task. Subsequent to the Route Direction Task, participants were asked to indicate the direction in which the route continued after movement stopped given the current travel direction. For route repetition trials, this involves indicating the direction required to follow the original route toward the end. In contrast, for route retracing trials this involves indicating the direction required to return to the start location while remaining on the route. With three possible movement directions and an equal amount of trials requiring a left, right, and straight responses, chance level for this task was $33.3 \%$.

- Landmark Sequence Task. Finally, participants were presented with an image depicting three of the landmarks on the route. Their task was to indicate which of these landmarks would be encountered next, if the current travel direction was maintained. Chance level for this task was 33.3\%.

Participants were instructed to respond as accurately and as quickly as possible by pressing the correspondingly labeled buttons on a response box (Cedrus RB-730).

The Route Direction Task, the Intersection Direction Task, and the Landmark Sequence Task were designed to test different aspects of route learning and route retracing. To solve the Route Direction Task participants needed to compare the sequence of landmarks and/or turns encountered during travel with their route memory. The Intersection Direction Task and the Landmark Sequence Task require participants to anticipate the upcoming movement direction or the next landmark, respectively. For repetition trials, these tasks are functionally equivalent to standard route learning tasks (Waller and Lippa, 2007; Head and Isom, 2010). Retracing trials, in contrast, require further processing: the Landmark Sequence Task requires manipulation of the temporal order of the landmarks; the Intersection Direction Task requires comprehending the spatial relationships between the direction from which an intersection is approached and the direction in which the route continues.

\section{ANALYSIS}

We first analyzed performance for the Route Direction Task. Only trials in which participants correctly identified travel direction were included in the analyses of the Intersection Direction and the Landmark Sequence Task. As a result of removing incorrect Travel Direction Task trials, fewer data points were available for the remaining analyses. In order to analyze effects of learning on these two tasks we, therefore, pooled data from sessions $1-3$ and $4-6$.

\section{RESULTS \\ ROUTE DIRECTION TASK}

In order to enter the final data-set, participants' performance in repetition trials on the Route Direction Task had to exceed chance level (50\%). This criterion was set to ensure that participants had acquired sufficient route knowledge during the training phases to investigate the effects of cognitive aging on route repetition. Performance for two older participants did not reach chance level. These participants were excluded from the final data-set.

To examine the impact of travel direction (route repetition and route retracing) on performance in the Route Direction Tasks between age groups, a repeated-measures ANOVA was conducted with the between-subject factors of age group (young, older) and sex and two within-subject factors of experimental session (1-6) and travel direction (repetition, retrace). We observed main effects of age $\left[F_{(1,34)}=9.74, p<0.01\right.$, $\left.\eta^{2}=0.22\right]$, session $\left[F_{(3.2,111.17)}=42.20, p<0.001, \eta^{2}=0.55\right]$, direction $\left[F_{(1,34)}=26.36, p<0.001, \eta^{2}=0.44\right]$, but not of sex $\left[F_{(1,34)}=0.16, p=0.69, \eta^{2}=0.01\right]$. Specifically, younger participants performed better than older participants $(86.19 \%$ vs. $74.97 \%$ ), performance on repetition trials was better than on retracing trials $(88.08 \%$ vs. $73.07 \%)$, and performance improved over experimental sessions (session 1: 60.96\%, session 6: 89.94\%).

The main effect of direction was primarily driven by the impaired performance of older participants on retracing trials (see Figure 2). This is reflected in the significant interaction of direction $x$ age group $\left[F_{(1,34)}=10.31, p<0.01, \eta^{2}=0.23\right]$ and by post-hoc tests demonstrating that younger and older participants performed comparatively during route repetition trials [t-test $(89.31 \%$ vs. $\left.87.25 \%): t_{(35.75)}=0.73 ; p=0.47\right]$, but performed differently during route retracing trials [ $t$-test $(84.27 \%$ vs. $\left.63.32 \%): t_{(23.50)}=3.59 ; p<0.01\right]$. Only one other interaction, direction $x$ session $\left[F_{(2.9,100.7)}=3.55, p=0.02, \eta^{2}=0.10\right]$ was significant.

Note that participants who entered this analysis were selected on basis of their performance on repetition trials. However, despite good performance on repetition trials, older adults showed impaired performance on retracing trials compared to young adults. In other words, the subsample of older adults that had no problems identifying travel direction during route repetition, exhibited a specific age-related impairment when traveling in the opposite direction along the route.

How did participants solve the Route Direction Task? In principle there are two ways: first, participants can compare the order in which the landmarks are encountered during training and test phase; second, for those test trials that feature a turn at the first intersection encountered, participants can compare the turning direction at this intersection between training and test. The analysis of response times for test trial with a turn at the first intersection allows us to distinguish between these two alternatives: Turning onset was $4.7 \mathrm{~s}$ after the test trial started. After $7.4 \mathrm{~s}$ the landmark of the second intersection was in sight. Response time for the relevant test trials was $10.25 \mathrm{~s}$ for young adults, $12.08 \mathrm{~s}$ for older adults, $11.06 \mathrm{~s}$ for repetition trials, and $11.26 \mathrm{~s}$ for retracing trials. There was a main effects of age $\left[F_{(1,36)}=8.33, p<0.01\right.$, $\left.\eta^{2}=0.19\right]$ but no main effect of direction $\left[F_{(1,36)}=1.91, p=\right.$ $\left.0.18, \eta^{2}=0.05\right]$ and no significant interaction $\left[F_{(1,36)}=1.52\right.$, $p=0.23, \eta^{2}=0.04$ ] (see Figure 2 right). Participants made their decision $3 \mathrm{~s}$ (younger) to $5 \mathrm{~s}$ (older) after the second landmark was in sight. This strongly suggests that participants analyzed the order in which landmarks were encountered during travel to inform their decision, rather than the turning direction at the first intersection encountered. 


\section{Route Direction Task}
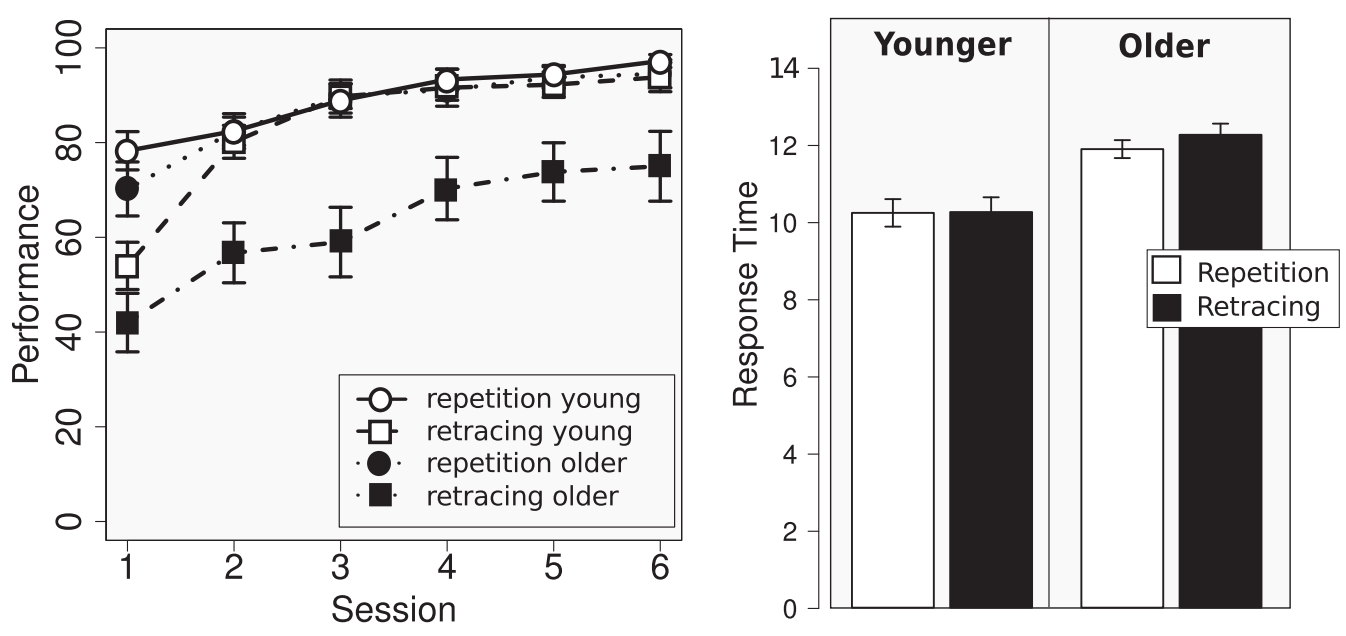

FIGURE 2 | Left: performance for the Route Direction Task; right: response time for test trials featuring a turn at the first intersection encountered.

\section{INTERSECTION DIRECTION TASK}

As a result of removing two older participants from the final dataset and of excluding data from incorrect Route Direction Task trials from the further analysis, fewer trials from older participants (1446) than from younger participants (1720) entered the analysis of the Intersection Direction Task and the Landmark Sequence Task.

In order to examine performance differences between age groups in the Intersection Direction Task, a repeated-measures ANOVA was conducted with the between-subject factor of age (younger, older) and two within-subject factors of experimental session (first and second half of the experiment) and movement direction (repetition, retrace). We observed main effects of session $\left[F_{(1,36)}=29.76, p<0.001, \eta^{2}=0.45\right]$, direction $\left[F_{(1,36)}=29.51, p<0.001, \eta^{2}=0.45\right]$, and age $\left[F_{(1,36)}=\right.$ 17.39, $\left.p<0.001, \eta^{2}=0.33\right]$. The main effect of age was driven both by repetition trials (post-hoc: $p<0.001$ ) and by retracing trials (post-hoc: $p<0.01$ ).

Of the interactions only direction $x$ session $\left[F_{(1,36)}=9.07\right.$, $\left.p<0.01, \eta^{2}=0.20\right]$ and direction $x$ session $x$ age group $\left[F_{(1,36)}=4.12, p=0.05, \eta^{2}=0.10\right]$ were significant. These interactions were primarily driven by impaired learning in the older adults in the retracing trials: while young participants' performance improved over the course of the experiment for both repetition and retracing trials, the older adults' performance improved only on repetition trials, but not on retracing trials (see Figure 3). This is corroborated by additional separate repeatedmeasures ANOVAs for the young and the old age group: while main effects of direction and session were observed for both age groups (all $p<0.05$ ), the interaction between direction and session was significant only in the older age group $(p=0.02)$, but not in the young age group $(p=0.12)$.

Note that performance for retracing trials in the older age group remained close to chance level performance (33\%) for the entire experiment (see Figure 3).

\section{LANDMARK SEQUENCE TASK}

In order to examine performance differences between age groups in the Landmark Sequence Task, a repeated-measures ANOVA was conducted with the between-subject factor of age (younger, older) and two within-subject factors of experimental session (first and second half of the experiment) and movement direction (repetition, retrace). We observed main effects of age $\left[F_{(1,36)}=11.54, p<0.01, \eta^{2}=0.24\right]$ and session $\left[F_{(1,36)}=95.07, p<0.001, \eta^{2}=0.73\right]$, but not of direction $\left[F_{(1,36)}=2.30, p=0.14, \eta^{2}=0.04\right]$. Overall, performance was better for the young age group (young: $76.20 \%$; old: $63.31 \%$ ) and increased over the course of the experiment (first half: 62.22\%; second half: $77.29 \%$; see Figure 3). While none of the interactions was significant, the direction $x$ age group interaction neared significance $\left[F_{(1,36)}=4.04, p=0.052\right.$, $\left.\eta^{2}=0.10\right]$.

\section{DISCUSSION}

In this study we used a novel experimental paradigm to investigate the effects of cognitive aging on the ability to retrace a route-i.e., to navigate from the end of a route back to the start location. In the experiment, participants first viewed a visual presentation of a route and were then presented with short segments of the route either in the direction experienced during training (route repetition trials) or in the opposite direction (route retracing trials). For each of these presentations, participants were given three tasks: in the Route Direction Task they had to indicate the overall travel direction; in the Intersection Direction Task, they had to indicate the direction of movement required to remain on the route given the current travel direction; in the Landmark Sequence Task they had to indicate which landmark they would encounter next when proceeding in the current travel direction.

Consistent with earlier findings (Barrash, 1994; Wilkniss et al., 1997; Moffat et al., 2001; Head and Isom, 2010), the older age 


\section{Intersection Direction Task}

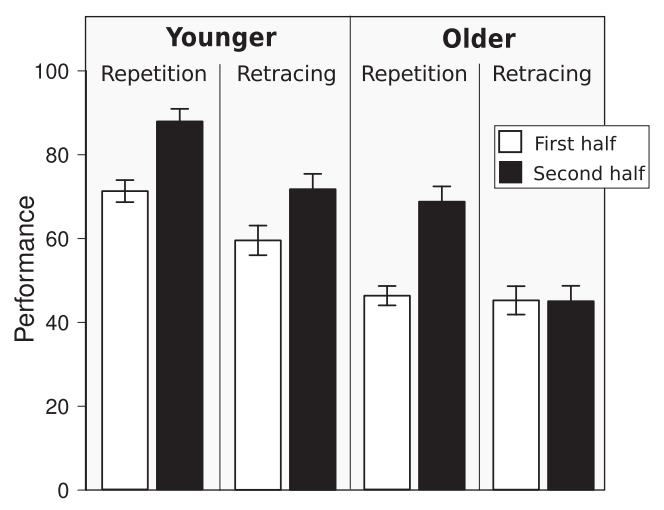

\section{Landmark Sequence Task}

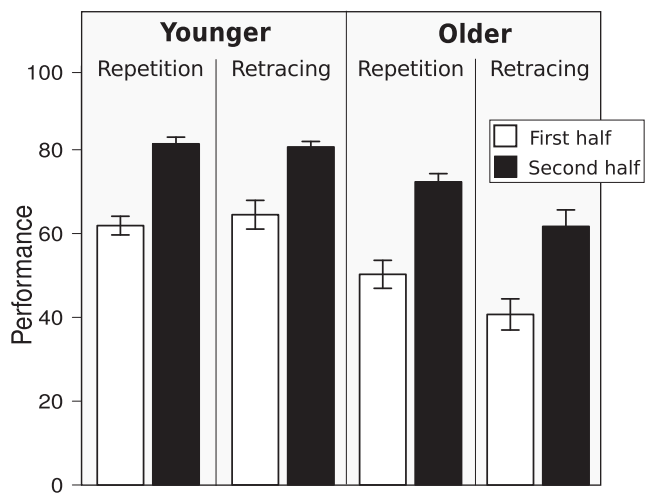

FIGURE 3 | Left: performance for the Intersection Direction Task; right: performancre in the Landmark Sequence Task.

group showed an overall performance deficit in route learning, even after removing two participants from the final data-set who did not reach chance level performance in route repetition trials in the Route Direction Task. In addition, older adults also showed specific deficits in retracing trials for both the Route Direction Task and the Intersection Direction Task. That is, older adults experienced difficulties when asked to identify the overall travel direction and to indicate the next movement direction when retracing the route, but not when repeating the route. Given the importance of successfully retracing a recently navigated route when exploring unfamiliar environments (Lorenz, 1952; Miller and Eilam, 2011), these effects offer an explanation for why age-related declines in navigation abilities are more striking in novel than in familiar environments (Devlin, 2001) and why older adults often report avoiding unfamiliar routes and places (Burns, 1999). In the following we consider results from the three tasks in the test phase in more detail.

\section{ROUTE DIRECTION TASK}

The Route Direction Task assessed participants' ability to identify the current travel direction (route repetition or route retrace) while being passively transported along a short segment of the route. While this task can, in principle, be solved by comparing the turning direction at a single intersection during test with that experienced during training, participants of both age groups only responded after they encountered a second landmark. This suggests that they used the temporal ordering of landmarks to solve the task. Both younger and older adults performed well on route repetition trials. In contrast, for route retracing trials we found performance decrements in the older age group. In other words, while the older adults that entered the final data-set were perfectly able to recognize travel direction when it was identical to that during training, they had problems doing so when travel direction was reversed. This may be explained by the involvement of different cognitive processes during route repetition and route retracing: in the Route Direction Task participants are asked to identify travel direction by matching the order of landmarks experienced during the test phase to that experienced during training. Research in the area of sequence processing suggests that in repetition trials this task recruits a supervisory process that involves monitoring and selectively activating relevant items while suppressing irrelevant ones (Oberauer et al., 2000). For retracing trials, however, the sequence is reversed thus additionally requiring the coordination of the relative positions between the items. The efficiency of this coordination process has been found to be affected by cognitive aging (Bopp and Verhaeghen, 2007) which could explain the specific impairment for retracing trials in the older age group.

\section{LANDMARK SEOUENCE TASK}

Older adults were also less accurate than young adults in the Landmark Sequence Task (see also Lipman and Caplan, 1992; Wilkniss et al., 1997; Head and Isom, 2010). However, the older adults reached performance levels clearly above chance level and performance increased over the course of the experiment. We did not observe a main effect of travel direction for the Landmark Sequence Task: both age groups performed at similar levels on repetition trials and on retrace trials. This seems surprising at first glance, but can be explained by (1) both the Route Direction Task and the Landmark Sequence Task requiring comparison between the temporal ordering of landmarks during the training phase with that experienced during the test phase; and (2) that only the data from correct Route Direction Task trials entered the Landmark Sequence Task analysis. In other words, as both tasks rely on similar processes and by pre-selecting trials on basis of performance in one task, potential effects of travel direction in the second task are reduced.

\section{INTERSECTION DIRECTION TASK}

In line with earlier research (e.g., Head and Isom, 2010), older adults were less accurate in the Intersection Direction Task than young adults. This effect was observed both for route repetition trials as well as for route retracing trials. In addition, we found a specific learning deficit in retracing trials for the older age 
group: performance in retracing trials was close to chance level and, in contrast to repetition trials and the younger age group, did not improve over the course of the experiment. That is to say, not only did older adults perform weaker in indicating the correct direction when retracing a route, they were also unable learn the correct direction over the course of the experiment.

Egocentric route learning strategies (Trullier et al., 1997; Waller and Lippa, 2007) enable a navigator to solve the Intersection Direction Task in route repetition trials. Route retracing, in contrast, is not supported by egocentric strategies as intersections are approached from a different direction than during training. Route retracing, therefore, requires abstracting from the viewpoint-dependent memory encoded during the learning phase. This can be achieved by encoding the spatial relationship between the arm from which a particular intersection was approached and the arm in which the route proceeded. This form of representation is independent of the navigator's viewpoint and, therefore, allocentric. Recent research suggests that age-related deficits in allocentric processing (Moffat and Resnick, 2002; Moffat et al., 2007; Harris and Wolbers, in press) result from hippocampal degeneration during typical aging (Raz et al., 2010; Wiener et al., under review). This could also explain the age-related decline in performance on route retracing trials as these, in contrast to the route repetition trials, require allocentric processing.

In addition to the proposed deficits in allocentric processing resulting from age-related hippocampal degeneration, more general age-related declines in working memory and processing speed may contribute to the observed effects. The Processing Speed Theory states that declines in processing speed with increasing age can result in impairments in cognitive performance (Salthouse, 1996). This may be due to task-related time limitations or because results of earlier cognitive operations are no longer available when later operations are completed (simultaneity). While the current paradigm did not impose time limitations, allocentric processing is computationally more demanding than egocentric processes as it requires additional cognitive operations (Byrne et al., 2007).

\section{REFERENCES}

Barnes, C. A., Nadel, L., and Honig, W. K. (1980). Spatial memory deficit in senescent rats. Can. J. Psychol. 34, 29-39.

Barrash, J. (1994). Age-related decline in route learning ability. Dev. Neuropsychol. 10, 189-201.

Begega, A., Cienfuegos, S., Rubio, S., Santin, J. L., Miranda, R., and Arias, J. L. (2001). Effects of ageing on allocentric and egocentric spatial strategies in the wistar rat. Behav. Processes 53, 75-85.

Bopp, K. L., and Verhaeghen, P. (2007). Age-related differences in control processes in verbal and visuospatial working memory: storage, transformation, supervision, and coordination. J. Gerontol. B Psychol. Sci. Soc. Sci. 62, 239-246.
Burgess, N. (2008). Spatial cognition and the brain. Ann. N.Y. Acad. Sci. 1124, 77-97.

Burns, P. C. (1999). Navigation and the mobility of older drivers. J. Gerontol. B Psychol. Sci. Soc. Sci. 54, 49-55.

Byrne, P., Becker, S., and Burgess, N. (2007). Remembering the past and imagining the future: a neural model of spatial memory and imagery. Psychol. Rev. 114, 340-375.

Colgin, L. L., and Moser, E. I. (2006). Neuroscience: rewinding the memory record. Nature 440, 615-617.

Devlin, A. (2001). Mind and Maze: Spatial Cognition and Environmental Behavior. Westport, CT: Praeger.

Foster, D. J., and Wilson, M. A. (2006). Reverse replay of behavioural
Any age-related processing speed effects are, therefore, more likely to affect retracing trials which rely on allocentric processing rather than repetition trials for which egocentric processes are sufficient.

We have argued that route retracing relies on allocentric processing. This is supported by a series of findings: route retracing provides a means to return to the start of a journey and has, therefore, been suggested to be crucial for exploring novel environments (Lorenz, 1952). Accordingly, in freely exploring rodents, route retracing is mainly observed during early stages of learning an environment (Miller and Eilam, 2011). Early stages of spatial learning primarily rely on allocentric place strategies, whereas egocentric response or route strategies only occur later (Tolman et al., 1946; Ritchie et al., 1950). Together with results from the current study this suggests that route retracing during the early stages of learning an environment relies on allocentric strategies.

Recent electrophysiological findings in rats suggest a neuronal mechanism that could support route retracing. Foster and Wilson (2006) recorded from hippocampal place cells and found that immediately after traversing a track, the hippocampal place cell activity observed during navigation was reactivated in reverse temporal order as if retracing the route. Importantly, these reverse replays were observed to a greater extent after navigating through a novel environment compared to a familiar environment, suggesting that such replays play an important role during spatial learning (see also Colgin and Moser, 2006).

To conclude, we have demonstrated age-related deficits in route retracing. Specifically, older participants showed impaired performance in both recognizing travel direction when navigating along the route in the reverse direction and in indicating the direction required to retrace the route. Given the importance of route retracing for learning novel environments these findings provide further insights into the effects of normal cognitive aging on wayfinding and orientation abilities.

\section{ACKNOWLEDGMENTS}

Many thanks to Gemma Hanson for her help in collecting data. sequences in hippocampal place cells during the awake state. Nature 440, 680-683.

Harris, M., and Wolbers, T. (in press). Ageing effects on path integration and landmark navigation. Hippocampus.

Head, D., and Isom, M. (2010). Age effects on wayfinding and route learning skills. Behav. Brain Res. 209, 49-58.

Iaria, G., Palermo, L., Committeri, G., and Barton, J. J. (2009). Age differences in the formation and use of cognitive maps. Behav. Brain Res. 196, 187-191.

Lipman, P. D., and Caplan, L. J. (1992). Adult age differences in memory for routes: effects of instruction and spatial diagram. Psychol. Aging 7, 435-442.
Lorenz, K. (1952). King Solomon's Ring - New Light on Animal Ways. New York, NY: Meridian Books (Penguin).

Luis, C. A., Keegan, A. P., and Mullan, M. (2009). Cross validation of the montreal cognitive assessment in community dwelling older adults residing in the southeastern US. Int. J. Geriatr. Psychiatry 24, 197-201.

McDonald, R. J., and White, N. M. (1994). Parallel information processing in the water maze: evidence for independent memory systems involving dorsal striatum and hippocampus. Behav. Neural Biol. 61, 260-270.

Miller, M., and Eilam, D. (2011) Decision making at a crossroad: why to go straight ahead, retrace a path, 
or turn sideways? Anim. Cogn. 14, 11-20.

Moffat, S. (2009). Aging and spatial navigation: what do we know and where do we go? Neuropsychol. Rev. 19, 478-489.

Moffat, S., Kennedy, K., Rodrigue, K., and Raz, N. (2007). Extrahippocampal contributions to age differences in human spatial navigation. Cereb. Cortex 17, 1274-1282.

Moffat, S., and Resnick, S. (2002). Effects of age on virtual environment place navigation and allocentric cognitive mapping. Behav. Neurosci. 116, 851-859.

Moffat, S., Zondermann, A., and Resnick, S. (2001). Age differences in spatial memory in a virtual environment navigation task. Neurobiol. Aging 22, 787-796.

Nasreddine, Z. S., Phillips, N. A., Bedirian, V., Charbonneau, S., Whitehead, V., Collin, I., Cummings, J. L., and Chertkow, H. (2005). The Montreal cognitive assessment, MoCA: a brief screening tool for mild cognitive impairment. J. Am. Geriatr. Soc. 53, 695-699.
Nicolle, M. M., Prescott, S., and Bizon, J. L. (2003). Emergence of a cue strategy preference on the water maze task in aged C57B6 x SJL F1 hybrid mice. Learn. Mem. 10, 520-524.

Oberauer, K., Suss, H., Schulze, R., Wilhelm, O., and Wittmann, W. (2000). Working memory capacity facets of a cognitive ability construct. Pers. Individ. Dif. 29, 1017-1045.

Raz, N., Ghisletta, P., Rodrigue, K. M., Kennedy, K. M., and Lindenberger, U. (2010). Trajectories of brain aging in middle-aged and older adults: regional and individual differences. Neuroimage 51, 501-511.

Ritchie, B., Aeschliman, B., and Pierce, P. (1950). Studies in spatial learning. viii. Place performance and the acquisition of place dispositions. J. Comp. Physiol. Psychol. 43, 73-85.

Rodgers, M. K., Sindone, J. A., and Moffat, S. D. (2012). Effects of age on navigation strategy. Neurobiol. Aging 33, 15-22.

Rosenzweig, E. S., and Barnes, C. A. (2003). Impact of aging on hippocampal function: plasticity, network dynamics, and cognition. Prog. Neurobiol. 69, 143-179.

Salthouse, T. A. (1996). The processingspeed theory of adult age differences in cognition. Psychol. Rev. 103, 403-428.

Tolman, E., Ritchie, B., and Kalish, D. (1946). Studies in spatial learning: place learning versus response learning. J. Exp. Psychol. 36, 221-229.

Trullier, O., Wiener, S. I., Berthoz, A. and Meyer, J. (1997). Biologically based artificial navigation systems: review and prospects. Prog. Neurobiol. 51, 483-544.

Waller, D., and Lippa, Y. (2007). Landmarks as beacons and associative cues: their role in route learning. Mem. Cognit. 35, 910-924.

Wilkniss, S. M., Jones, M. G., Korol, D. L., Gold, P. E., and Manning, C. A. (1997). Age-related differences in an ecologically based study of route learning. Psychol. Aging 12, 372-375.

Wolbers, T., Weiller, C., and Buchel, C. (2004). Neural foundations of emerging route knowledge in complex spatial environments. Brain Res. Cogn. Brain Res. 21, 401-411.

Conflict of Interest Statement: The authors declare that the research was conducted in the absence of any commercial or financial relationships that could be construed as a potential conflict of interest.

Received: 30 March 2012; accepted: 06 May 2012; published online: 21 May 2012.

Citation: Wiener JM, Kmecova $H$ and de Condappa O (2012) Route repetition and route retracing: effects of cognitive aging. Front. Ag. Neurosci. 4:7. doi: 10.3389/fnagi.2012.00007

Copyright (C) 2012 Wiener, Kmecova and de Condappa. This is an openaccess article distributed under the terms of the Creative Commons Attribution Non Commercial License, which permits non-commercial use, distribution, and reproduction in other forums, provided the original authors and source are credited. 